\title{
A DESIRABLE CONVULSIVE THRESHOLD. SOME REFLECTIONS ABOUT ELECTROCONVULSIVE THERAPY (ECT)
}

\author{
Emiliano Loria \\ Sapienza - University of Rome \\ Review article - Received: 17/8/2020 Accepted: 5/10/2020
}

\begin{abstract}
Long-standing psychiatric practice confirms the pervasive use of pharmacological therapies for treating severe mental disorders. In many circumstances, drugs constitute the best allies of psychotherapeutic interventions. A robust scientific literature is oriented on finding the best strategies to improve therapeutic efficacy through different modes and timing of combined interventions. Nevertheless, we are far from triumphal therapeutic success. Despite the advances made by neuropsychiatry, this medical discipline remains lacking in terms of diagnostic and prognostic capabilities when compared to other branches of medicine. An ethical principle remains as the guidance of therapeutic interventions: improving the quality of life for patients. Unfortunately, psychotropic drugs and psychotherapies do not always result in an efficient remission of symptoms. In this paper I corroborate the idea that therapists should provide drugresistant patients with every effective and available treatment, even if some of such interventions could be invasive, like Electroconvulsive Therapy (ECT). ECT carries upon its shoulders a long and dramatic history that should be better investigated to provide new insights. In fact, ECT has attracted renewed interest in recent years. This is due to the fact that antidepressant drugs in younger patients show often scarce effectiveness and unpleasant side-effects. Moreover, I show that, thanks to modern advances, ECT may work as a successful form of treatment for specific and rare cases, such as severe depression (with suicide attempts) and catatonia.
\end{abstract}

Keywords: ECT; neuroendocrinology; psychopharmacology; history of child psychiatry 


\begin{abstract}
"The story of psychiatry is a story of tensions that have not yet played out. It is a history where what we decide is the truth regarding our past has immediate and profound implications for how we view ourselves and our futures and how we treat others when they are at their most vulnerable. It is a history that affects all of us."
\end{abstract}

(Healy 2002, 8)

\title{
1. The History of a Euphoria
}

Electroconvulsive therapy has an almost centennial history that began in Rome (Italy) in 1938, at the Clinic of Nervous and Mental Diseases, run at the time by (psychiatrist) Ugo Cerletti. ECT still represents one of the most important and controversial therapeutic discoveries in the field of psychiatry. It is based on the passage of short electrical impulses that cause convulsions. Unlike insulin, and still more than the drug Metrazol used by von Meduna to provoke convulsions but with severe side effects (Shorter and Healy 2007), electricity could be administered to patients in subtly graded doses. This way, the convulsions, which were considered by psychiatrists to be the essential therapeutic ingredient, would be less dangerous and easier to manage (Passione 2004, 89).

After the first official presentation of electroshock (E.S., as ECT was called at that time) to a restricted audience, Cerletti waited two years before the publication of a detailed account of his research in the Rivista sperimentale di freniatria (Cerletti 1940). Cerletti was probably aware of the potential risks involved in the dissemination of ECT, and he wanted to avoid hasty imitators applying E.S. with too little experience. He also believed that publicity about his discovery would have sounded inappropriate before a thorough analysis of "the best modes of application of E.S." was completed (Cerletti 1940). The long and detailed essays by Bini (1940) "La Tecnica e le manifestazioni dell'Elettroshock", testify that the inventors of this technique, namely Cerletti and Bini himself, were extremely careful and cautious to carry out electroconvulsive treatment (Passione 2004, 90-91).

However, their advice was not followed. The rapid dissemination of ECT in Western countries was due to Lothar Kalinowsky, a Jewish student of Cerletti emigrated from Italy to England because of the racial laws promulgated in 1938 during the Fascist regime. The application of the therapy occurred in every conceivable way (Berrios 1997; Shorter 1997). From England to France, to Germany and to the United States, where another collaborator of Cerletti, Renato Almansi, taught the E.S. technique to David Impastato, who treated the first American patient, a 29-year-old schizophrenic woman in 1940 (Passione 2004, 93). 
At the XXIII Congress of the Italian Society of Psychiatry held in 1946, Cerletti denounced the non-scientific use of the recent shock therapeutic discoveries. In the same congress, psychiatrists Baldi and Reale raised the same question about the indiscriminate abuse of shock therapies, in particular ECT, for all types of mental illness. They posited the need to "arrive at a clearer definition of its field of application and restrict its use accordingly" (Baldi and Reale 1947; Passione 2004, 96). Max Fink, one of the most famous American psychiatrists who practiced and taught ECT throughout his long career, has well described these initial phases in an interview with Shorter and Healy in 2002. He claimed: "The problem at the beginning was what is necessary for an effect. It was [easier] to get seizures. But they didn't know what was important, so they tried sub convulsive, they tried convulsive, they tried multiple seizures, they tried different electrode pairs, with drugs, without drugs, daily treatments, twice daily" (Shorter and Healy 2007, 140).

As patient and clinical reports attest, it was not uncommon for E.S. to be administered without a real therapeutic need and in combination with insulin therapy and psychotropic drugs. ${ }^{1}$ In some cases, E.S. was unfortunately used as a deterrent or even as a threat. These repeated occurrences contributed to the creation of stigma surrounding ECT and to its wrongful assimilation to a barbaric practice.

Every catatonic or schizophrenic patient was the ideal candidate, together with adolescents and children. The first minor patients treated with ECT were believed to have been in France starting in 1941 by George Heuyer and colleagues, and in the United States starting in 1942 by Lauretta Bender (see Shorter 2013). ${ }^{2}$ However, from the examination of the Archives of Pediatric Neuropsychiatry in Rome - a section of the Roman Clinic specifically dedicated to minors ${ }^{3}$ - it emerges that the first child ever administered with ECT (September 18, 1940) was a 7-year-old boy diagnosed with "dementia praecocissima". ${ }^{4}$ After 23 applications of the technique, the child was discharged and returned to the father, who had assisted him during the preparation of the first session. Another child (a

\footnotetext{
${ }^{1}$ For the Italian case, see Nemec (2015) who discusses several clinical reports and treatments relative to the post-WWII era in Trieste and Gorizia.

${ }^{2}$ See also Walter et al. (2010).

${ }^{3}$ The hospital section devoted to minors was established by the Director Sante de Sanctis in 1930 (Coccanari de' Fornari et al. 2017).

${ }^{4}$ Historical Archives of Child Neuropsychiatry - Department of Human Neuroscience (Sapienza University of Rome), volume n. 18, record 1682. The ordering of the medical records which are in the Historical Archives follows an arrangement by years. For a review about childhood dementias according to the diagnostic criteria of that time, see Campailla (1945, 264-265).
} 
six-year-old boy) with the same diagnosis was treated with E.S. in 1943. Before and after them, a few other children and adolescents were treated with insulin shock therapies. The first catatonic young patient treated with E.S. was a 14-year-old girl in 1944. After 11 applications, the girl had a remission of symptoms and was discharged from the clinic and returned to her family. She relapsed years later and was readmitted to the clinic in December 1950. Accornero and Anderson (1948) reported a sample of E.S. treatments which regarded infant and young adolescent patients of the Roman clinic. Among these cases is also the first one I mentioned. ${ }^{5}$ Compared to the original medical record, the article by Accornero and Anderson does not provide many other data. In general, the medical records of those years often report a detailed anamnesis. However, the outcome of the treatments is hard to obtain. Accornero and Anderson's essay has the merit of deepening this aspect for some of the 15 cases of prepubertal schizophrenia they examined. This way, we come to discover that Gianluigi, this is the name of the first child, is discharged from the Clinic in the same conditions in which he entered. Furthermore, the frequency of treatments is well specified in the paper (the first 12 applications twice a week and the other ones three times a week). The authors also specify the standard voltage: 110 or 120 Volt in $1 / 10$ per second (Accornero and Anderson 1948, 233-234). Accornero and Anderson's conclusive analysis is pessimistic about the efficacy of ECT in childhood psychosis. The authors were aware of the small sample of patients, and mightily complained of the difficulty in providing a more detailed diagnosis. The vagueness of the diagnosis increased the empiricity of the therapeutic method which was already empirical.

The archives of the Roman Clinic of Nervous and Mental Diseases also bring to light the existence of an informed consent form that families (or caretakers) were asked to sign, albeit in a primitive form. In the E.S. Collection, established by the Director Cerletti, there are the primitive informed consent forms that the parents, relatives or tutors had to fill in. Initially, in 1937, the informed consent only concerned insulin therapy and it had later been extended to ECT. It recited as follows:

[On letterhead R.[oyal] University of Rome. Clinic of Nervous and Mental Diseases] I have been made aware of the dangers that shock therapies pose in some cases. Given that these treatments are today those that give some hope for improvement, I adhere to the proposal to practice it in my son

\footnotetext{
${ }^{5}$ It is worth noting that Felice Accornero helped Cerletti and Bini in making the ECT device (Sirgiovanni and Aruta 2020, 321). Thus, two remarkable researchers had already reported the very first case of E.S. in children, although they did not underline this peculiarity.
} 
[or other family member] [name and surname of the patient], releasing the R[oyal] Clinic from any responsibility. [Original signature.$^{6}$

Nowadays, patients are routinely told why a certain treatment is prescribed, and the administration procedures are explained in detail. At the time, of course, each patient had to sign informed consent forms where all this information was illustrated in writing. The family members also had to agree.

\section{From Euphoria to Barbarities}

From its beginning in 1938 throughout the 1950s, ECT gained wide fame for the treatment of schizophrenia. Those who have the opportunity to consult old medical records dating back to those years may realize the series of therapeutic success, partial symptomatic remissions, cyclic relapses, and consequent readmissions, as well as complete failures of ECT. Although this scenario cannot be considered a full-fledged victory, the therapeutic success attained was already an exceptional improvement, which could illuminate the darkness of therapeutic nihilism in which psychiatry as a science had been struggling for centuries.

The ECT practice also revealed its efficacy for catatonia and affective disorders like severe depression. In a very few years, from the era of medical frustration where there was no other remedy than to sedate, purge and contain patients, and "the treatment of mental illness represented a vast wasteland of hopelessness" (Shorter and Healy 2007, 4), ECT appeared to provide fast alleviation of psychotic symptoms, above all in the acutely ill, without too many significant risks (see below for a discussion on ECT's side effects).

Throughout the 1960s and 1970s, the wide availability and dissemination of antipsychotic drugs, together with increasing opposition to the use of ECT, that went into a gradual decline (Fink 2001). During the years of contestations, i.e. from 1968 onward, a destructive as well as misleading connection was generated between the movements of psychiatry renovation and antipsychiatry. The former worked on changing the methods of interventions and limiting the restrictive measures adopted in asylums. The latter was against both the asylums and psychiatric interventions as a whole, including shock therapies and drugs. Drugs, which had not much earlier been acclaimed "as awakening the chronically

\footnotetext{
${ }^{6}$ My translation is from original documents, which are kept in the E.S. Collection.
} 
psychotic from irretrievable madness, were now castigated as chemical straitjackets" (Healy 2002, 5). All of psychiatry's past was interpreted as a series of barbarities culminated in E.S. and psychosurgery. Similarly, for many philosophers at that time, the methods of psychiatric discipline represented a clear and potent symbol of the irrationality of modern society, generated by the union between science and capitalism. ${ }^{7}$

This political and cultural short circuit led to two dramatic changes, first of all in Italy, which acted as a pioneering country in this field. The first was the closure of psychiatric asylums, which in many cases had become institutions of arbitrary and unmotivated restraints (see Babini 2009 on the Italian case). The second was the abandonment of those therapeutic practices that were considered too invasive. This way, psychiatric treatments of proven effectiveness such as E.S. were banned, little by little, in many (Western) countries (Healy 2002, 5). A resurgence of interest in ECT occurred in the 1980s, following the finding that a large portion of patients do not tolerate or respond to drugs, or appear to be drug resistant. This has prompted the investigation of combining drugs with ECT. Furthermore, many psychiatrists suggest ECT as the treatment of choice for catatonic schizophrenia (Fear 2005, 30).

\section{Barefaced and Raw ECT}

Given these historical premises, we cannot disagree with Edward Shorter when he claims that ECT provides an exciting insight into the role of culture against science in clinical decision-making (Shorter 2013). Why should we reconsider ECT after a massive and historically-laden disengagement from this therapy? The answer is simple: ECT is an effective and safe treatment, with potentially low side-effects risks for some kinds of severe disorders. Quoting the UK National Institute for Clinical Excellence (NICE): "Electroconvulsive therapy is a proven effective treatment for depression. It is a safe form of treatment even in the medically ill, the elderly and in pregnancy. There are benefits in using it in emergencies. It should not be relegated to a treatment of last resort" (Lamprecht et al. 2005, 19). In fact, ECT could be the treatment of choice when severe forms of depression are associated with attempts of suicide (or with strong suicidal plans) and urgent intervention is needed, or when patients present significant psychomotor retardation, stupor, depressive

\footnotetext{
${ }^{7}$ I suggest reading Giovanni Jervis on this point (2014). He was a lucid witness of the cultural climate in Europe and also contributed to a profound renewal of psychiatric interventions in Italy. For more details see also Fiorani's article (this issue).
} 
delusions or hallucinations (Lamprecht et al. 2005, 13; see also UK ECT Review Group 2003).

Contrary to their original hopes (and promises), some kinds of psychotropic drugs like selective serotonin reuptake inhibitors (SSRIs) have been accompanied by several distressing side effects. Among them, decreased libido and even impotence, delayed orgasm, anorgasmia, anhedonia. Impotence has proven particularly troublesome, as Clayton and colleagues showed (2002; but see also Jacobsen et al. 2020). Furthermore, twenty years ago in the UK, two-thirds of SSRI patients had discontinued treatment by the end of the third month (Shorter 2009, 201). ${ }^{8}$

When pharmacotherapy fails to improve depressive symptoms, then, "response rates of about 50-60\% can be achieved by ECT" (Eser et al. 2007, 2). For this reason, particularly in depressed patients at high risk of suicide, ECT should be recommended earlier than its conventional "last resort" position. In fact, the risks of suicide have been shown to relieve quickly through ECT, when administered in continuity with previous treatments, that are essential to sustain its benefits. Look, for example, at Kellner et al.'s $(2005,977)$ study, in which expressed suicidal intent in 131 depressed patients was rapidly relieved by ECT, already after one week (i.e., three ECT sessions) in $38.2 \%$ of the patients, and, at the end of the course of treatment, in $80.9 \%$ of the patients.

Furthermore, in all stages of pregnancy, ECT may represent the best solution in case of severe mood or thought disorders, when antidepressants and antipsychotics cannot be administered (Abrams 2002; Fink 2009). In this sense, ECT may represent a safer treatment for these more difficult cases.

In addition to drug-resistant major depression, which remains the most frequent indication for ECT, other psychiatric illnesses like delusional mania, catatonia, malignant neuroleptic syndrome and delusional mania should be considered "urgent first-line indications for ECT treatment" (Eser et al. 2007, 2). Patient choice is obviously crucial in most cases. In fact, if some sufferers have already experienced ineffective or intolerable medical treatment, they may choose ECT; alternatively, if they had previous successful experiences of recovery with ECT, they can express the will to be administered a further cycle of treatment. ${ }^{9}$ Although ECT has

\footnotetext{
${ }^{8}$ For a review of these issues, see Holtzheimer and Mayberg (2011).

${ }^{9}$ Several handbooks and essays about psychiatry insert positive biographical reports about ECT treatments (see references below). In this regard, see the recent collection edited by
} 
been recognized as "an effective treatment for mood and psychotic disorders, it is among patients with catatonia that the most remarkable efficacy is observed" (Petrides et al. 2004, 151). Although for uncomplicated cases of catatonia the first-line treatment should be benzodiazepines (Rosebush and Mazurek 2004), "for malignant catatonia, or in circumstances where use of benzodiazepines is contraindicated, ECT should be the first-line treatment" (Fear 2005, 40). On the contrary, ECT is not helpful for those people affected by a lifelong history of emotional dysfunctions, or suffering of personality disorders, addictions, sociopathy (Fink 2009).

\section{The Procedure}

The image of a fast, shift-like psychiatry is far from the truth: "ECT is not a surgical excision that removes the defect", claims Fink. "It is more like the treatment of diabetes in which repeated administration of insulin and dietary control are needed to maintain healthy serum glucose levels" (Fink 2013, 21).

Thanks to the evolution of ECT instrumentation, which often includes $\mathrm{EEG}^{10}$ and EKG (for a review see Weiss 2018, 16-18), the current procedure involves the use of brief-pulse stimulation techniques which induct a series of generalized epileptic seizures under anesthesia and muscle relaxation. This procedure is "one of the best tolerated and safest biological treatment strategies with low risk for severe complications" (Eser et al. 2007, 2; see also Abrams 2002). A typical course of ECT treatment consists of two or three inductions a week for two to seven weeks (Fink 2009, 4). Fink explains the procedure as follows:

The patient is asked to empty the bladder and is then taken to the treatment room, where she lies down on a stretcher. A nurse or physician inserts a needle into a vein in the arm or foot, attaches a bottle of fluid (usually sugar in water), and sets the fluid flowing at a slow rate. [...] This intravenous line allows the easy and painless administration of medications during the treatment. Adhesive monitoring electrodes - flat, disposable pads or reusable discs to which electrical connections can be

Kirov (2020). In the Bini archive, there are letters from patients containing thanks to Lucio Bini and Ugo Cerletti for the improvement of the conditions after the E.S. treatments. (Passione 2007, 89)

10 "The EEG facilitates discrimination between electrode positions and different stimulus doses. [...] EEG may indicate threshold changes highlighting the need to increase the stimulus dose or reduce the anesthetic induction agent" (Weiss 2018, 17). 
made - are applied to the skin, a painless procedure. Three electrodes are put in place for the electroencephalogram (EEG); two stimulating electrodes for the electrical stimulus; three for the electrocardiogram (ECG) and heart rate; and two to measure motor movements during treatment. A recording electrode placed on the patient's finger or toe measures the blood oxygen saturation. A blood pressure cuff on the arm measures the blood pressure, and a second one may be placed as a tourniquet on a leg to allow the psychiatrist to record the duration of the muscular signs of the seizure. (Fink 2009, 14)

During the seizure, the medical team needs to monitor heart rate and rhythm, blood pressure and the brain's electrical activity. Some dental conditions require a personalized plastic braces for each treatment.

Electrodes placement affects the procedure: treatments based on bilateral electrode placement usually exhibit shorter duration. The successful use of bilateral ECT is reported, for example, for severe forms of catatonia. I provide more details on this below. Another important issue is the combination of ECT therapy with antipsychotics. Since ECT is not considered as therapy of first choice in catatonia, patients often come to ECT after benzodiazepines administration or while they are receiving this medication. In these cases, a drastic withdrawal is not recommended, because it may cause worsening or relapse of the most severe symptoms. Indeed, a synergic effect of ECT and benzodiazepines has been reported by Petrides and colleagues, who also claim that "benzodiazepines are often useful and can be continued for many months after recovery" (Petrides et al. 2004, 156-157; Weiss 2018, 70-71).

\section{The Underlying Mechanism of ECT: A Neuroendocrinological Perspective}

An objective and scientific-based reason that can explain why ECT, by giving patients epileptic-like seizures, should be beneficial to some psychiatric illness remains quite obscure. What we know is that adrenocorticotropic hormone (ACTH), thyrotropin releasing hormone (TRH), and vasopressin are well-studied hormones that are known to be released by seizures. The amount of hormones released varies with electrode placement, stimulation dosage, number and frequency of treatments. Prolactin, for example, is quickly released within the serum with a peak at 20 minutes. According to Fink, "it is the massive outpouring of these hormones that characterize the seizures in effective ECT" (Fink 2013, 21). 
As it is known, the hypothalamus plays a crucial role in sustaining life because it regulates the autonomic nervous and neuroendocrine systems. We also know that hormonal functions in mental illness are disordered. For instance, in severe depressive states, the axis linking hypothalamus, pituitary gland, and adrenal and thyroid glands appears to be dysfunctional, involving increases of cortisol and other hormones. ECT with bitemporal electrode placement allows the passage of electricity from one temple to the other, and as a result the hypothalamus is directly stimulated. The first effects of such stimulation are transitory, but by the fourth or fifth stimulus - according to Max Fink- "the normal feedback actions of the hormones of the hypothalamic-pituitary-adrenal axis are again in place. Feeding and sleep become normal, and improvements in motor activity, mood, memory, and thought to follow quickly" (Fink 2013, 24). It is no coincidence that ECT is most effective in patients who present neuroendocrine dysfunctions (e.g. "abnormal dexamethasone suppression test or diminished thyroid-stimulating hormone response to thyrotropinreleasing hormone"), which can disappear with effective treatment (Petrides et al. 2004, 158).

However, the neurotransmitter pathway has also been explored to better understand the ECT effects. For what concerns depression, several studies show that ECT can attenuate serotonergic and noradrenergic neurotransmission (for a review see Eser et al. 2007, 3). Two phenomena have been observed in this respect. On the one hand, ECT would increase the availability of the serotonin precursor thus contributing to the therapeutic effects of ECT (Palmio et al. 2005). On the other hand, in line with the GABA deficit hypothesis of depression, ECT would exert a compensatory increase in $\gamma$-aminobutyric acid (GABA) neurotransmission. For instance, a study based on proton magnetic resonance spectroscopy showed that "occipital cortex GABA concentrations are increased in depressed patients treated with ECT" (Eser et al. 2007, 3; see also Sanacora et al. 2003).

The behavioral effects of seizures require repetition until the pathological symptoms have remitted, and new patterns of behavior have stabilized. The prescription of antidepressants after a successful ECT administration reduces the risk of early relapse (Weiss 2018). As I mention above, antidepressants should not be suddenly discontinued before ECT, especially those with a short half-life or SSRIs. Those patients previously medicated with SSRIs should be initially administered "a low electrical dose at the first treatment" (Scott 2005, 111). 


\section{Side-effects}

Currently, the mortality rate of ECT is estimated at two deaths per 100,000 treatments. The causes of death referring to adults during ECT are from cardiovascular disorders, most often pre-existing conditions, such as cardiac arrhythmia or hypertension, when these fail to be taken into account by clinical practitioners and anaesthesiologists. According to proton magnetic resonance spectroscopy studies, it seems that neuronal damage or cell death are not induced by ECT, because it does not provoke a significant decrease in the $\mathrm{N}$-acetylaspartate signal, which constitutes a sign of cell atrophy (Ende et al. 2000; Eser et al. 2007, 4).

No deaths have been ever reported in an adolescent or a child directly due to ECT. Mortality associated with ECT has markedly decreased with technological improvement and medical monitoring during the applications. For this reason, nowadays, ECT can be considered a low-risk procedure, "even among older patients with cardiac disorders" (Consoli et al. 2013, 141). Anaesthesia may obviously provoke other side effects, including disturbance in cardiac rhythm, variations in blood pressure, respiratory incidents, and allergic reactions. Less serious side effects of ECT include headache and muscle pain, nausea (with or without vomiting), temporary confusion immediately after the sessions, and understandable fear about ECT. "Up to $45 \%$ of patients report headache after ECT, which can be treated using analgesics such as acetylsalicylic acid or paracetamol and, if severe, by changing the induction medications" (Eser et al. 2007, 10). Even if rarely, nausea may occur after intravenous anesthesia; in this case metoclopramide can be used successfully. Since ECT is not a firstline treatment, when psychiatrists suggest or prescribe ECT, patients often present a very compromised medical status. When ECT is early administered in the course of catatonia and in severe forms of depression, then more favourable results and reduced morbidity are obtained. Furthermore, such severe psychiatric disorders often entail cognitive impairments per se. Thus, it is challenging to evaluate eventual cognitive damages due to ECT or to illness.

In the last twenty years, an increasing number of studies have focused on ECT effects on memory. The meta-analysis conducted by Fraser and colleagues (Fraser et al. 2008) suggests that ECT may cause autobiographical memory impairment. Still, such memory loss is relatively short-term, i.e., about six months post-treatment. The potential cognitive deficits refer to autobiographical episodes and events that occurred in the period close to the beginning of treatment. Furthermore, implicit memory, procedural memory and semantic memory are not modified by ECT (Consoli et al. 2013). Therefore, it is reasonable to claim that memory loss 
due to ECT is temporary and circumscribed to some particular events close to the sessions. The degree of these temporary memory damages depends on many factors such as the number of sessions, the features of the electrical current (e.g. brief pulsed or sinusoidal), the placement of the electrodes, and the presence of persistent depressive symptoms before ECT (Sackeim et al. 1991). In brief, variations in the administration methodology may affect cognitive function after ECT. From randomized studies, it seems that "sinusoidal waveform causes more memory impairment than brief-pulse ECT" (Lamprecht et al. 2005, 18), and, for this reason, brief-pulse stimulation techniques represent the most common stimulation method nowadays.

This brief overview shows how 80 years after the invention and introduction of ECT, the methodology of its application has improved both to avoid collateral physical damage and to enhance the effectiveness of treatment according to the type and severity of the disease. ${ }^{11}$

\section{Electrode placement}

Given the importance of lateralization and, more generally, of the allocation of the electrodes for what concerns efficacy and risks of ECT, some further considerations are necessary. First, it is established that "stimulus intensity depends on electrode placement" (Eser et al. 2007, 5). In this sense, bilateral ECT seems to be more effective than unilateral (UL) ECT, which requires a higher stimulus dosage to gain the same results. Many practitioners doubt the effectiveness of UL ECT in treating patients who have severe or life-threatening psychopathologies (Weiss 2018). Consequently, the final choice of the more effective positioning of the electrodes requires a balance of costs and benefits for the suffering patients. The electrode placement, in fact, constitutes a crucial factor for cognitive side effects; in this regard, it seems that bifrontal electrode placement is as efficacious as bitemporal placement in inducing less severe cognitive impairment (Bailine et al. 2000). ${ }^{12}$ The UK ECT Review Group recommends that bilateral arrangement is preferred when rapid and complete recovery has priority, whereas UL is preferred when minimizing cognitive adverse effects has priority.

When treatment is not urgent, an initial trial of unilateral ECT will significantly shift the cost-benefit balance because of the

\footnotetext{
11 It is worth noting that the World Health Organization promoted the ban on unmodified ECT (Leiknes et al. 2012).

12 Notably, in schizophrenic patients the influence of electrode placement (or stimulus intensity) is less obvious than in depressive patients (Eser et al. 2007).
} 
substantial reduction in the risk of severe or persistent retrograde amnesia. [...] Clinical monitoring of symptoms and possible cognitive adverse effects is necessary in any case throughout treatment, and a lack of satisfactory improvement may lead to an increase in the electrical dose or a switch to bilateral electrode placement if there had been no clinical improvement. (Scott 2005, 135-136)

What is the optimal frequency of treatments? According to Scott (2005, 141), in the case of bilateral treatment, the optimal frequency is twice per week, which may be reduced if cognitive adverse effects emerge. A three times per week administration could be justified only in severe, lifethreating depression, since it leads to a faster reduction of depressive symptoms, but "at the cost of more pronounced cognitive adverse effects" (Scott 2005, 140). For what concerns UL ECT, the optimal frequency seems to be twice per week.

\section{ECT in Pediatric Care}

The stigma of ETC is even more evident when talking about its applications in children and adolescents. Any public and professional discourse over ECT in minors is likely "to be emotive, rhetorical, and unbalanced" (Robertson et al. 2013, 59). A (young) person receiving ECT is liable to experience stigma because of his or her age, illness, and treatment. It is a fact that negative attitudes toward ECT contribute to this stigma, which entails negative consequences such as concealment of illness and social exclusion (McDonald and Walter 2013, 51). However, when people who hold these prejudices encounter individuals who have received ECT, or gain accurate information about the treatment, they view the treatment in a more positive light. As Fink claims, "patients undergoing ECT have proved to be its best advocates" $(2009,11)$. It is the case of young patients and their parents as the studies of Walter et al. (1999) and Flamerique et al. (2017) show. In both studies, the researchers used a selfadministered questionnaire to assess the experience, knowledge, and attitudes of parents of adolescents who had been treated with ECT about the treatment. In the former, 28 parents were interviewed and expressed favorable opinions about ECT: 17 of 28 parents claimed that ECT had been helpful. The latter investigated parents of adolescents (under the age of 18) diagnosed with schizophrenia and treated with ECT. They were compared with a randomly selected group of parents of adolescents treated with drugs. 
Most parents in the ECT group claimed that they had received adequate information about the ECT procedure (94.7\%), most of them thought it had been helpful for their children (73.7\%) and none thought that it had made things worse. The large majority of parents in the ECT group (80\%) thought that the illness had been worse than ECT or medication, and none thought that ECT was the worst. (Flamerique et al. 2017, 1)

In adolescence, ${ }^{13}$ treatment-resistant mood disorder constitutes a severe debilitating illness which may be successfully treated with ECT when treatment resistance is well identified (Ghazziudin 2013). Depression and catatonia in children and adolescents constitute a severe and debilitating disorder that can be life-threatening, and even in less severe cases, it significantly affects the quality of life of patients and their families. Furthermore, these kinds of disorders negatively affect normal growth and development. The treatment of prepubescent children still occurs in rare cases, while for adolescents the scientific literature confirms that prescriptions, safety and efficacy are the same as for adults (Fink 2009, Abrams 2002; Fink and Taylor 2003; Cohen et al. 2000; Consoli et al. 2012).

Nevertheless, there is a gap in the guidelines of many national institutes when this unpleasant failure of treatment occurs. For example, the American Academy of Child and Adolescent Psychiatry Practice Parameters for the assessment and treatment of children and adolescents with depression do not include ECT as a treatment option. The first-line treatment of moderate to severe adolescent depression is usually SSRI combined with evidence-based psychotherapy (CBT or IPT). Failure to respond to one or two SSRIs is often followed by treatment with a noradrenergic antidepressant. Ghazziudin estimates that "approximately $71 \%$ of adolescents may respond to initial combination treatment (fluoxetine and CBT) by the end of a 12-week period, although remission rates may be higher when treatment is administered over a longer duration" (Ghazziudin 2013, 185). It is worth noting that antipsychotic agents often have substantial side effects, such as weight gain and obesity associated with an elevated risk for diabetes mellitus (Holt 2019). Additionally, an increased risk of suicide rates has been associated with Antiepileptic drugs (AEDs), that can function as mood stabilizers and are often used for augmenting antidepressants. Still, they result in a "black box" warning by the US Food and Drug Administration (FDA). Ghazziudin found that "one in every four or five adolescents diagnosed with MDD may not respond to two sequentially used antidepressant agents administered inadequate dose

${ }^{13} \mathrm{ECT}$ is an extremely rare procedure in children below 12 years of age. 
and duration" $(2013,177)$. Some of these young patients might reasonably benefit from ECT.

\section{ECT side effects in adolescents}

ECT is usually well-tolerated by many adolescents. Some studies reported in Consoli et al. $(2008,155)$ indicate that "most former adolescent recipients report a positive experience and attitude toward ECT", despite initial and understandable apprehension. The possible presence of cognitive impairments among adolescents treated by ECT has been analyzed by Ghazziudin and colleagues (2000):

Comparison of pre-ECT and the first post-ECT testing administered during the first 10 days of the treatment yielded significant impairments of concentration and attention, verbaland visual-delayed recall, and verbal fluency. A complete recovery of these functions was noted at the second post-ECT testing. There was no deficit in the ability to problem solve during the initial or the subsequent testing. (Ghazziudin et al. 2000, 269, italics added)

Although caution is in order while we wait for further confirmation from larger samples, these results attest the evidence already emerged among adults: there is no long-term injury in terms of concentration, attention, visual memory, and verbal fluency. Side effects of ECT in young persons are generally transient, as in the adult population. As David Cohen, director of the child and adolescent psychiatry service at the Pitié-Salpêtrière hospital group in Paris, claimed: "there is no ethical reason to ban ECT use in adolescents". On the contrary, "unrealistic fears regarding ECT" lead to untreated minors, even in cases of dramatic conditions (Cohen et al. 2000, $1)$.

\section{Legal and Ethical Concerns}

In the USA, despite the updated recommendations of the American Psychiatric Association (APA) for ECT practice, the legislative framework concerning ECT differs among the member States. For example, in California and Texas there is a stricter legislation than the norms recommended by the APA. The EU boasts the creation of a European Forum for Electroconvulsive Therapy (EFFECT - founded in 2005) ${ }^{14}$ and

14 "EFFECT brought together clinicians and researchers to improve the practice of ECT, and to reduce its stigma" (https://www.theeffect.eu/). 
a Task Force on ECT within the World Federation for Societies of Biological Psychiatry (WFSBP). Nevertheless, there is no unified European recommendation on ECT yet (Robertson et al. 2013). In Slovenia and Luxembourg, ECT is not available to treat people of any age. In Romania people are sometimes treated without anaesthesia because anaesthesiologists are not always available, while in Spain, Austria, Slovakia, Greece, Switzerland, and Germany, ECT is available and separate consent for anaesthesia is required (Gazdag et al. 2012, 6). In Italy, Ireland, and Latvia a written informative consent is needed for each ECT session, while in Portugal and the UK, one informative consent is valid for a definite number of sessions (12 or 15) (Gazdag et al. 2012).

ECT is so stigmatized that its use is severely limited, and its merits are neglected or even denied. The numerous attacks that ECT has received are often supported by ideological reasons not based on science or clinical experience. Nevertheless, these attacks have obtained political attention and often led to legislative restrictions (Ottosson and Fink 2004, 19). Misconceptions regarding ECT are associated with negative attitudes toward it, but prejudices and folk beliefs should not be involved in the scientific, ethical, and political discourse. Psychiatry - as Shorter and Fink note - "has swung wildly from fashion to fashion from asylum care to psychoanalysis to lobotomy to psychopharmacology without having an underlying scientific rationale for doing so. More than any other medical field, psychiatry has been guided by cultural preferences and political persuasions" (Shorter and Fink 2010, vi). It is time to "regroup" and adopt a "multifaceted approach" to change this bleak picture (McDonald and Walter 2013, 51). We must surrender to the idea that there is a group of psychiatric patients who are drug-resistant, or they cannot assume drugs for several reasons. In a nutshell, the ethical puzzle that I raise is the following. Is it possible to administer a therapy to help these suffering patients (be them adults or minors)? If the answer is yes, at least for some types of severe diseases, why should we refrain from administering that therapy? Why should we ignore, for ideological reasons, robust therapeutic possibilities? Why should we prolong the severity of the symptoms by making the life of patients and their family members unbearable? One therapy for the improvement of some severe psychotic symptoms exists and is practicable. ECT is such a therapy. In this sense, ECT does not constitute an alternative model of treatment, but an additional therapeutic tool that does not replace, but rather integrates pharmacotherapy and psychotherapy.

In conclusion, it is worth noting that the last decades have seen the promising development of several invasive treatments for brain stimulation aimed to relieve severe neuropsychological impairments in neurological 
and psychiatric patients, like for example Transcranial Magnetic Stimulation (TMS), and Deep Brain Stimulation (DBS) (see Pycroft et al. 2018 for a review). If ECT is a well-established treatment for depression and catatonia, DBS is applied in patients who have severe Parkinson's disease, as well as depression (Marcolin and Padberg 2007, vii). Many of these brain stimulation techniques "converge in terms of underlying mechanisms of action based on fundamental principles of brain function" and differentiate each other in virtue of specific characteristics regarding the levels of invasiveness and the duration of intervention (Ibid., viii). For example, DBS, is, de facto, a permanent stimulator into the brain and it represents "a long-term treatment, particularly suitable for chronic or frequently relapsing disorders" (Ibid.). These novel methods find great attention without triggering reprobation, while ECT is still condemned.

\section{Conclusion}

Electroconvulsive Therapy represents an appropriate treatment for severely ill psychiatric patients who need hospital care. In those Western countries where ECT is permitted and practiced, it is usually recommended after long, unsuccessful courses of psychotherapies and psychotropic drugs. To summarize in a nutshell the current practice, I follow Yuval Bloch's words, according to whom the major reason for child and adolescents referrals to ECT is represented by the severity of symptoms, particularly catatonia and suicidal behaviour, while the major reason for referral of adults is the long and persistent failure of response to pharmacotherapy (Bloch et al. 2008). In this paper I set out to show that a further step is possible to promptly provide appropriate and effective care in those difficult cases. However, a cultural change is necessary to get started with this process.

\section{Acknowledgments}

I would like to thank Valentina Petrolini, Marko Jurjako, and Matteo Fiorani, excellent travel companions.

\section{REFERENCES}

Abrams, R. 2002. Electroconvulsive Therapy. New York: Oxford University Press.

Accornero, F., and M. Anderson. 1948. L'elettroshock nella psichiatria infantile. Il Lavoro Neuropsichiatrico 3(2): 231-250. 
American Psychiatric Association. 2001. Electroconvulsive Therapy: Recommendations for Treatment, Training and Privileging Washington: APA.

Babini, V. 2009. Liberi Tutti. Manicomi e Psichiatri in Italia: Una Storia del Novecento. Bologna: Il Mulino.

Bailine, S. H., A. Rifkin, E. Kayne, J. A. Selzer, J. Vital-Herne, M. Blieka, and S. Pollack. 2000. Comparison of bifrontal and bitemporal ECT for major depression. American Journal of Psychiatry 157, 121-123.

Baldi, F., and G. Reale. 1947. L'elettroshock nella terapia neuropsichiatrica. Rivista Sperimentale di Freniatria 72: 239-42.

Berrios, G. E. 1997. The scientific origins of electroconvulsive therapy: A conceptual history. History of Psychiatry 8: 105-119.

Bini, L. 1940. La tecnica e le manifestazioni dell'elettroshock. Rivista Sperimentale di Freniatria 18: 361-458.

Bloch Y., D. Sobol, and Y. Levkovitz. 2008. Reasons for referral for electroconvulsive therapy: A comparison between adolescents and adults. Australasian Psychiatry 16: 191-194.

Campailla, G. 1945. Le demenze infanto-prepuberali. Archivio di Psicologia Neurologia Psichiatria e Psicoterapia 4(4): 262-338.

Cerletti, U. 1940. L'elettroshock. Rivista Sperimentale di Freniatria 18: 209-310.

Clayton, A. H., J. F. Pradko, H. A. Croft. et al. 2002. Prevalence of sexual dysfunction among newer antidepressants. Journal of Clinical Psychiatry 63(4): 357-366.

Coccanari de' Fornari, M. A., A. Iannitelli, and M. Biondi. 2017. Storia della clinica psichiatrica della Sapienza Università di Roma nel policlinico Umberto I. Rivista di Psichiatria 52(1): 1-8.

Cohen, J., M. Flament, O. Taieb, C. Thompson, and M. Basquin. 2000. Electroconvulsive therapy in adolescence. European Child \& Adolescent Psychiatry 9: 1-6.

Consoli, A., J. Cohen, N. Bodeau, V. Guinchat, and L. Wachtel. Electroconvulsive therapy in adolescents with intellectual disability and severe self-injurious behavior and aggression: A retrospective study. European Child \& Adolescent Psychiatry 22(1): 55-62.

Consoli, A., W. De Carvalho, and D. Cohen. 2013. Side effects of ECT. In Electroconvulsive Therapy in Children and Adolescents, eds. N. Ghazziudin and G. Walter, pp. 140-160. New York: Oxford University Press.

Ende, G., D. F. Braus, S. Walter, W. Weber-Fahr, and F. A. Henn. 2000. The hippocampus in patients treated with electroconvulsive therapy: A proton magnetic resonance spectroscopic imaging study. Archives of General Psychiatry 57, 937-943. 
Eser, D., C. Schüle, R. Rupprecht, and T. C. Baghai. 2007. Electroconvulsive therapy: Update and new research. In Transcranial Brain Stimulation for Treatment of Psychiatric Disorders, eds. M. A. Marcolin and F. Padberg. Advances in Biological Psychiatry 23: 1-17.

Fear, C. F. 2005. The use of ECT in the treatment of schizophrenia and catatonia. In The ECT Handbook, ed. A. Scott, pp. 30-46. London: The Royal College of Psychiatrists

Fink, M., and M. A. Taylor. 2003. Catatonia: A Clinician's Guide to Diagnosis and Treatment. Cambridge: Cambridge University Press.

Fink, M. 2011. Transcranial magnetic stimulation is not a replacement for electroconvulsive therapy in depressive mood disorders. Journal of ECT 27: 3-4.

Fink, M. 2013. The mechanism of action of ECT. In Electroconvulsive Therapy in Children and Adolescents, eds. N. Ghazziudin, G. Walter, pp. 18-28. New York: Oxford University Press.

Fiorani, M. this issue. Rationality, irrationality and irrationalism in the anti-institutional debate in psychiatry around the second half of the 1970s in Italy. European Journal of Analytic Philosophy. https://doi.org/10.31820/ejap.16.2.4

Flamarique, I., I. Baeza, E. de la Serna, A. Pons, M. Bernardo, and J. Castro-Forniele. 2017. Thinking about electroconvulsive therapy: The opinions of parents of adolescents with schizophrenia spectrum disorders. Journal of Child and Adolescent Psychopharmacology 27(1): 75-82.

Fraser, L. M., R. W. O'Carroll, and K. P. Ebmeier. 2008. The effect of electroconvulsive therapy on autobiographical memory: A systematic review. The Journal of ECT 24(1): 10-17.

Ghazziudin, N. 2013. ECT for Mood Disorder. In Electroconvulsive Therapy in Children and Adolescents, eds. N. Ghanzi and G. Walter, pp. 161-190. Oxford University Press.

Ghazziudin, N., D. Laughrin, and B. Giordani. 2000. Cognitive side effects of electroconvulsive therapy in adolescents. Journal of Child and Adolescent Psychopharmachology 10(4): 269-276.

Gazdag, G., R. Takács, G. Ungvari, and P. Sienaert. 2012. The practice of consenting to electroconvulsive therapy in the European Union. Journal of ECT 28(1): 4-6.

Healy, D. 2002. The Creation of Psychopharmacology. Cambridge: Harvard University Press.

Heuyer G., and F. Bour. 1942. Electro-choc chez les adolescents. Annales Médico-Psychologiques 2: 75-84.

Hoekstra, R., W. W. van den Broek, D. Fekkes, J. A. Bruijn, P. G. Mulder, and L. Pepplinkhuizen. 2001. Effect of electroconvulsive therapy 
on biopterin and large neutral amino acids in severe, medication resistant depression. Psychiatry Research 103, 115-123.

Holt, R. I. G. 2019. Association between antipsychotic medication use and diabetes. Current Diabetes Reports 19(96) doi: 10.1007/s11892019-1220-8.

Holtzheimer, P. E., and H. S. Mayberg. 2011. Stuck in a rut: Rethinking depression and its treatment. Trends in Neuroscience 34(1): 1-9.

Jacobsen, P. L., E. M. Thorley, and C. Curran. 2020. Real-world patient experience with sexual dysfunction and antidepressant use in patients with self-reported depression: Across-sectional survey study. Neurology, Psychiatry and Brain Research 36: 57-64.

Jervis, G. 2014. Contro il Sentito Dire. Psicoanalisi, psichiatria e politica. Torino: Bollati Boringhieri.

Kellner, C. H., M. Fink, R. Knapp, G. Petrides, T. Rummens, M. Mueller, H. Bernstein, K. Rasmussen, K. O'Connor, G. Smith, A. J. Rush, M. Biggs, S. McClintock, S. Bailine, and C. Malur. 2005. Relief of expressed suicidal intent by ECT: A consortium for research in ECT study. American Journal of Psychiatry 162, 977-982.

Kirov, G. 2020. Shocked: Insider stories about electroconvulsive therapy. Amazon Kindle Direct Publishing.

Lamprecht, H. C., I. N. Ferrier, and A. G. Swann. 2005. The use of ECT in depressive illness. In The ECT Handbook, ed. Scott, A. I. p. 924. London: The Royal College of Psychiatrists.

Leiknes, K. A., L. J.-von Schweder, B. Høie. 2012. Contemporary use and practice of electroconvulsive therapy worldwide. Brain and Behavior 2(3): 283-344.

Marcolin M. A., and F. Padberg. 2007. Transcranial brain stimulation for treatment of psychiatric disorders. Advances in Biological Psychiatry 23.

Maayan, R., Y. Yagorowski, D. Gruppe, M. Weiss, B. Shtai, M. A. Kant, and M. A. Weizman. 2000. Basal plasma dehydroepiandrosterone sulfate level: A possible predictor for response to electroconvulsive therapy in depressed psychotic inpatients. Biological Psychiatry 48, 693-701.

McDonald, A., and G. Walter. 2013. ECT and Stigma. In Electroconvulsive Therapy in Children and Adolescents, eds. N. Ghanzi and G. Walter, pp. 29-55. USA: Oxford University Press.

Nemec, G. 2015. Dopo Venuti a Trieste. Storie di Euli Giuliano-dalmati attraverso un manicomio di confine 1945-1970. Trieste: Alphabeta verlag.

Ottoson, J. O., and M. Fink. 2004. Ethics in Electroconvulsive Therapy. New York: Brunner-Routledge (Taylor \& Francis). 
Passione, R. 2004. Italian psychiatry in an international context: Ugo Cerletti and the case of electroshock. History of Psychiatry 15(1): 83-104.

Passione, R. 2007. Ugo Cerletti. Il romanzo dell'elettroshock. Reggio Emilia: Aliberti.

Petrides, G., C. Malur, and M. Fink. 2004. Convulsive therapy. In Catatonia. From Psychopathology to Neurobiology, eds. S. N. Caroff, S. C. Mann, A. Francis, and G. L. Fricchione, pp. 151160. Washington: American Psychiatry Publishing.

Palmio, J., M. Huuhka, P. Saransaari, S. S. Oja, J. Peltola, E. Leinonen, J. Suhonen, and T. Keranen. 2005. Changes in plasma amino acids after electroconvulsive therapy of depressed patients. Psychiatry Research 137, 183-190.

Pycroft, L., J. Stein, and A. Tipu. 2018. Deep brain stimulation: An overview of history, methods, and future developments. Brain and Neuroscience Advances 2: 1-6.

Robertson, M., J. M. Rey, and W. G. Walter. 2013. Ethical and Consent Aspects. In Electroconvulsive Therapy in Children and Adolescents, eds N. Ghazziudin and G. Walter, pp. 56-75. New York: Oxford University Press.

Rosebush, M. F., and P. I. Mazurek. 2004. Pharmacotherapy. In Catatonia. From Psychopathology to Neurobiology, eds. Caroff, S. N., Mann, S. C., A. Francis, and G. L. Fricchione, pp. 141-150. Washington: American Psychiatry Publishing.

Sachs M., and V. Madaan. 2012. Electroconvulsive Therapy in Children and Adolescents: Brief Overview and Ethical Issues. Charlottesville: University of Virginia Health System.

Sackeim, H. A., Devanand, D. P., and J. Prudic. 1991. Stimulus intensity, seizure threshold, and seizure duration: Impact on the efficacy and safety of electroconvulsive therapy. Psychiatric Clinics of North America 14: 803-843.

Sanacora, G., G. F. Mason, D. L. Rothman, F. Hyder, J. J. Ciarcia, R. B. Ostroff, R. M. Berman, and J. H. Krystal. 2003. Increased cortical GABA concentrations in depressed patients receiving ECT. American Journal of Psychiatry 160, 577-579.

Sirgiovanni, E., and A. Aruta. 2020. The electroshock triangle: Disputes about the ECT apparatus prototype and its display in the 1960s. History of Psychiatry 31(3): 311-324.

Scott, A. I. F. ed. 2005. The ECT Handbook. The Third Report of the Royal College of Psychiatrists' Special Committee on ECT. London: The Royal College of Psychiatrists.

Shorter, E. 1997. A History of Psychiatry. From the Era of the Asylum to the age of Prozac. New York: John Wiley \& Sons. 
Shorter, E. 2009. Before Prozac. The Troubled History of Mood Disorders in Psychiatry. Oxford: Oxford University Press.

Shorter, E. 2013. The history of pediatric ECT. In Electroconvulsive Therapy in Children and Adolescents, N. Ghazziudin and G. Walter pp. 1-17. New York: Oxford University Press.

Shorter, E., and M. Fink. 2010. Endocrine Psychiatry: Solving the Riddle of Melancholia. New York: Oxford University Press.

Shorter, E., and D. Heale. 2007. Shock Therapy. A History of Electroconvulsive Treatment in Mental Illness. New Brunswick: Rutgers University Press.

UK ECT Review Group. 2003. Efficacy and safety of electroconvulsive therapy in depressive disorders: A systematic review and metaanalysis. Lancet 361, 799-808.

Walter, G., K. Koster, and J. Rey. 1999. Views about treatment among parents of adolescents who received electroconvulsive therapy. Psychiatric Service 50: 701-702.

Walter, G., M. Robertson, J. M. Rey, N. Soh, and G. S. Malhi. 2010. Electroconvulsive therapy in young people and the pioneering spirit of Lauretta Bender. Acta Neuropsychiatrica 22(5): 253254.

Weiss, A. 2018. The Electroconvulsive Therapy Workbook. Clinical Applications. London-New York: Routledge. 